\title{
Literatur und Kinderfilm
}

\section{Zur Situation in der Bundesrepublik Deutschland}

\author{
von Anton Täubl
}

Kinder und Jugendliche sind den audiovisuellen Medien in besonderer Weise ausgesetzt und werden dadurch von einem ständig wirksamen Einfluß betroffen, den diese "geheimen Miterzieher" ${ }^{\text {“1 }}$ ausüben. Lebensausschnitte und Verhaltensmuster, Wissen aller Art und spannende Unterhaltung kommen über Hörfunk, Bildschirm und Filmleinwand zu den Heranwachsenden. Die „vermittelte Erfahrung“, das „Leben aus zweiter Hand“ ist jedenfalls zu einem wichtigen Bestandteil ihres Lebens geworden. Die Verlängerung des Gehörsinnes und Sehvermögens durch das elektronische Informations- und Unterhaltungsangebot hat zunächst zu einer enormen Ausweitung der Wahrnehmung und zu einem zumindest vordergründigen Bewußtsein allerlei Zeitströmungen geführt.

Die tägliche Flut von Ton und Bild ist aber auch dabei, Kinder und Jugendliche zu überfordern. Bei der zunehmenden Menge und unterschiedlichen Art der Nachrichten und Eindrücke ist der Heranwachsende nicht mehr in der Lage, Informationen zu verarbeiten und zu ordnen. Die Fülle von Zahlen, Fakten und Meinungen, oft gegensätzlicher Art, orientieren nicht mehr, sondern desorientieren eher. Dadurch wird aber das unkritische Konsumverhalten begünstigt. Der Einzelne, der nicht gerlernt hat, die auf ihn aus verschiedenen Quellen zukommenden Informationen und Stellungnahmen zu vergleichen und abzuwägen, gerät damit in die Gefahr, Opfer der Manipulation zu werden: statt eines wirklichen Wissens von den Zuständen, tritt häufig ein verzerrtes und unvollständiges Bild der Wirklichkeit in den Vordergrund. In ihren Untersuchungen kommt die US-amerikanische Medienpädagogin Marie Winn zu dem pessimistischen Schluß, daß Heranwachsende durch übermäßigen Fernsehkonsum konzentrationsunfähig werden und kreatives Sprechen wie selbständiges Lesen verlernen bzw. daß sie Schwierigkeiten haben, es richtig zu lernen².

Von vielen betrauert wird in dem Zusammenhang aber auch ein Verlust an Phantasie, den ein überzogener Bilderkonsum angeblich verursache. Denn im Gegensatz zu Fernsehen und Filmeschauen „ist Lesen keine passive Rezeption, sondern aktive Koproduktion, eine selbständige mündige Leistung. Weil innerhalb des Textes viele Realisationen möglich sind, verdienen sie als Verstehensvarianten Toleranz, sofern sie sich belegbar im Text bewegen“3. "Verfilmung" hebe dagegen die Toleranz auf und fixiere den Betrachter auf Szenen, indem sie ihn durch fotographische Genauigkeit festlege und damit in seinen Vorstellungen auch einige. Film ist eben nur eine von vielen Möglichkeiten der Interpretation einer Partitur, ein „Abbild aus zweiter Hand“, nicht mehr ursprüngliche, sondern „vermittelte“ Realität. Der Film reduziere die „offene“ Lebenswirklichkeit auf eine Sicht, nämlich auf die des Autors bzw. des Regisseurs und Kameramanns.

Anton Täubl, von 1975 bis 1989 Produktionsreferent für katholische Religion beim Institut für Film und Bild in Wissenschaft und Unterricht, ist Geschäftsführer des Verlages steyl medien international, München. 
Auf dem Hintergrund dieser Geisteshaltung wird dann auch folgendes Urteil verständlich, das - obgleich aus dem Jahr 1917 stammend - viele Gemüter in der Bundesrepublik nach wie vor beschäftigt: „Der Märchenfilm nimmt vorweg, was sich in der Seele des Kindes (beim Lesen oder Erzählen - Anmerkung des Autors) abspielen sollte. Er hemmt die Phantasie und hebt damit eine im besonderen Maße dem Märchen vorbehaltene Funktion auf: Weckung der Phantasie $^{" 4}$. So verwundert die klagende Feststellung von Willy Wohlrabe, dem Seniorchef des Berliner "Jugendfilm-Verleihs“, denn nicht mehr: „Immer wieder müssen wir die Erfahrung machen, daß Lehrer Märchenfilme prinzipiell ablehnen, weil sie angeblich 'phantasieeinengend' wirken" ${ }^{\text {"5 }}$.

Gefürchtet und bedauert wird schließlich auch eine mit der Dominanz neuer Medientechnologien einhergehende Straffung der Arbeitsvorgänge und die damit gekoppelten Simplifizierung des Denkens. Die Tendenz geht ja zweifellos dahin, zumindest in Zeitungs- und Zeitschriften-Redaktionen, soweit wie möglich, die „papierlose Textverarbeitung“ einzuführen, das heißt, Redigieren von Texten auf dem elektronischen Bildschirm, gekoppelt mit einem direkten, durch nichts unterbrochenen Fluß vom Autor zur Endherstellung, zum Umbruch oder gleich zur Druckmaschine.

\section{Über Lesefreunde und Filmbesuch in der Bundesrepublik}

Aufschluß über den Medienkonsum der jüngeren Deutschen gibt die Untersuchung "Jugend und Medien" von den Rundfunkanstalten ARD und ZDF gemeinsam mit der Bertelsmann-Stiftung 1986 veröffentlicht. Intensiv befragt in Interviews wurden 149 Jugendliche und eine Gruppe von Eltern. Für die übliche Repräsentativ-Umfrage standen 4.000 Jugendliche zwischen 12 und 29 Jahre zur Verfügung. Gefragt wurde nach der Medien-Nutzung, also nach Fernsehen, Hörfunk, Tonträgern (z. B. Schallplatten, Musikkassetten usw.), Video, Zeitung, Zeitschrift und Buch.

Das Buch, dem bei Jugendlichen allgemein keine große Chance eingeräumt wird, kommt besser weg als gedacht! Zwar hat im fraglichen Zeitraum einerseits jeder fünfte Jugendliche kein Buch gelesen, andererseits liest aber jeder fünfte Jugendliche doch auch wieder "gerne" Bücher: Sie verbringen damit 1 Stunde und 40 Minuten am Tag. In der Gruppe der Jüngeren (12 bis 13 Jahre) lesen noch $28 \%$ regelmäßig Bücher und sie wenden dafür etwa 25 Minuten pro Tag auf. Dabei steht im literarischen Bereich die Unterhaltung nicht an erster Stelle. Fach- und Sachbücher, Lexika und Schulbücher bestimmen den täglichen Bedarf. In der Belletristik belegen Humor und Saire, Krimi- und Spionageliteratur die vorderen Ränge. Für die ganz Kleinen wurde das Erzählen wieder entdeckt. Anregungen hierzu geben oft reich bebilderte Broschüren, mal phantastisch, mal realistisch orientiert.

Die Zeitung, die für aktuelle Information wichtig ist, kommt nach dieser Umfrage bei den Jüngeren nicht gut weg. Das Fernsehen spielt nach wie vor eine große Rolle, vor allem bei der jüngeren Generation. Die 12 bis 15 jährigen sehen immerhin im Schnitt 2 Stunden pro Tag fern. Das Fernsehen erweist sich für die befragten Jugendlichen insgesamt als ein Medium der Unterhaltung, der 
aktuellen Information und im besonderen auch der Musik. Von den gängigen und beliebten Spiel- und Kinofilmen, spezifischen Jugend- und Musiksendungen wird mehr gewünscht; Sendungen aus Politik und Wirtschaft stoßen dagegen auf wenig Gegenliebe. Fernsehen gehört auch zur Alltagserfahrung von Klein- und Grundschulkindern. Der Erstkontakt liegt bei 2 Jahren; bereits $60 \%$ der 3jährigen sehen fern, nicht fernsehende 4jährige sind die Ausnahme.

Aufschluß über den Kinobesuch im Jahre 1986 gibt eine Statistik der Filmförderungsanstalt ${ }^{6}$ : Danach wurden 105,2 Millionen Kinobesucher in den 3.323 bundeseutschen Filmtheatern gezählt (1986 gab es noch 3.404 Kinos in der Bundesrepublik). Hauptbesuchergruppe bilden nach wie vor die 20 bis 29jährigen mit $42 \%$, gefolgt von den 14 bis 19 jährigen mit $39 \%$. Damit bestätigt sich die bekannte Größe und Struktur des Kinopublikums in der Bundesrepublik: Rund 2 Millionen Besucher pro Woche - bei 61 Millionen Einwohnern und davon über $80 \%$ im Alter zwischen 14 und 29 Jahren?. Der mit Abstand erfolgreichste Spielfilm war mit gut 6 Millionen Besuchern „Der Name der Rose“, eine Verfilmung des gleichnamigen Romans des italienischen Historikers Umberto Eco über Leben und Geist des Mittelalters. Es folgen die Komödie "Männer" von Doris Dörrie mit 4,8 Millionen Besuchern, „Momo“ von Johannes Schaaf nach der gleichnamigen phantastischen Erzählung von Michael Ende mit 2,1 Millionen; Margarethe von Trottas zeitpolitischen Frauenfilm „Rosa Luxemburg" haben bis Ende 1986 immerhin auch schon 519.000 Zuschauer gesehen.

In diesem Zusammenhang ist für den Bereich der Bundesrepublik allerdings noch ein weiterer, gerade für Kinder- und Jugendfilmarbeit ganz wichtiger Aspekt zu beachten: Neben der gewerblichen Vorführung von Filmen in den kommerziellen Kinos gibt es darüber hinaus auch zahlreiche nichtgewerbliche Spielstellen, die ohne Gewinnabsicht mit 16-mm-Filmen arbeiten. Es sind Kinos in Bürgerzentren, Jugendstätten, Kindergärten, Kulturhäusern und kirchlichen Gemeinden, sowie in Einrichtungen im Umfeld von Gewerkschaften, Parteien, Organisationen und Verbänden. Zu ihnen gehören eigens gestaltete Filmprogramme für Kinder und Jugendliche; oft entwickeln sie sich sogar zu speziellen Kinderkinos. Auch wenn noch keine exakten Zahlen dazu vorliegen, so gibt es nach Auskunft von Horst Schäfer, dem Leiter des Kinder- und Jugendfilmzentrums in der Bundesrepublik Deutschland, Remscheid, doch etwa 7.000 Abspielstellen und etwa 650 Verleihstellen über die gesamte Bundesrepublik verstreut $^{8}$. Stellt man diesen Hintergrund in Rechnung, so verwundert es nicht, daß der Film von Hero Senft „Ein Tag mit dem Wind" von 1978 weitaus mehr Zuschauer in nichtgewerblichen Filmtheatern erreicht hat, als in den kommerziellen Kinos. Das gleiche gilt für "Flußfahrt mit Huhn“ (Arend Agthe, 1983), der zu den erfolgreichsten Filmen der letzten Jahre in der Bundesrepublik Deutschland zählt: Innerhalb von 5 Jahren erreichte er rund 1 Million Zuschauer im nichtgewerblichen 16-mm-Bereich; im selben Zeitraum hatte dieser Film in der kommerziellen Auswertung lediglich knapp 100.000 Besucher!

\section{Quantitative Aspekte}

Wie sieht das Kinojahr in Zahlen aus? Im folgenden beziehe ich mich auf eine Analyse der Fachzeitschrift „film-dienst" für das Jahr 1986: 
- Insgesamt wurden 556 Filme erfaßt, die 1986 erstmals in der Bundesrepublik zur Aufführung gekommen sind (1985: 461 Filme),

- davon wurden 262 im Kino gezeigt (im Vorjahr 1985: 346),

- 194 Filme waren Erstaufführungen auf Video (1985: 115),

- erstmals im Fernsehen zu sehen waren 4 Kinofilme (1985: 13).

Bermerkenswert in unserem Zusammenhang ist, daß die Hälfte aller im „filmdienst" erfaßten Filme $(277=51 \%)$ nur für Erwachsene geeignet erschienen.

Als für Kinder bis 10 Jahre geeignet konnten lediglich 27 Filme (4,9\%) gewertet werden, darunter auch „Neues von uns Kindern aus Bullerbü“ (Schweden 1987, Regie: Lasse Hellström; Buch: Astrid Lindgren) nach einer Erzählung von Astrid Lindgren. 15 Filme (2,7\%) wurden ab 12 Jahre, 55 Filme (9,9\%) ab 14 Jahre und 119 Filme $(21,4 \%)$ ab 16 Jahre als geeignet eingestuft.

Über die Hälfte aller erfaßten Filme kamen aus den USA: 286 (1985: 234); darunter befanden sich 12 Koproduktionen mit anderen Ländern. Besonders deutlich ist die Dominanz der US-Produktionen im Video-Bereich festzustellen: 147 US-amerikanischen Videofilmen stehen 47 Filme aus anderen Ländern gegenüber; darunter 9 aus Kanada, 7 aus Italien, 6 aus England, 4 aus Australien, 3 aus Frankreich und nur 1 reine deutsche aus der Bundesrepublik (zu der sich noch 3 weitere Koproduktionen gesellen). Der Anteil der bundesdeutschen Filme am Kinoangebot hat sich nur gering verändert: 67 im Jahre 1986 gegenüber $69 \mathrm{im}$ Jahr 1985; darunter befinden sich 17 Gemeinschaftsproduktionen mit der CSSR, Finnland, Frankreich, Italien, Österreich, der Schweiz und Ungarn. 20 Filme stammen aus Frankreich, 19 aus England, 9 aus Italien, 7 aus der DDR, 5 aus Hongkong, jeweils 4 kamen aus Australien und der Schweiz; weiterhin kamen Filme auch aus Argentinien und Mexiko in die bundesdeutschen Kinos.

Die Anzahl der im Fernsehen ausgestrahlten Filme ist weiter gestiegen, was vor allem auf die kommerziell-orientierten Privatsender zurückzuführen ist. In der Beilage „Film im Fernsehen“ (Kath. Institut für Medieninformation e.V., Köln, 1987) wurden 1987 insgesamt 3.371 Filme im Fernsehen erfaßt: die öffentlich-rechtlichen Anstalten (ARD, ZDF, Dritte Programme) strahlten 1.990 Filme aus (Vorjahr 1985: 1.608), die privaten Fernsehsender wie RTL-plus oder SAT 1 zeigten 1.381 Filme (1985: 1.022 ohne Eins plus). Insgesamt befanden sich unter diesen im Fernsehen vorgeführten Filmen 432 Erstaufführungen, davon 293 in den öffentlich-rechtlichen Fernsehanstalten (1985: 265) und 139 in den privaten Fernsehprogrammen (Vorjahr: 96 ohne Eins plus).

Zahlreiche als Kinofilme bezeichnete Erstaufführungen waren Produktionen, die das Fernsehen gänzlich finanziert oder mitfinanziert hat. Ohne Beteiligung des Fernsehens an der Filmproduktion geht (fast) nichts mehr in der Filmbranche.

Was konnten Kinder in München an den schulfreien Tagen zwischen Weihnachten und Drei König (24.12.1988 bis 06.01.1989) im Kino und Fernsehen sehen? Was wurde ihnen an spezifischen Programmen über die Weihnachtsfeiertage angeboten? Im Kino gab es zu sehen ${ }^{10}$ : 
- „Die Abenteuer des Baron Münchhausen“, nach den Erzählungen von Rudolf Erich Raspe, ein Film von Terry Gilliam, nicht zu verwechseln mit einem UFAFilm über Baron Münchhausen aus dem Jahre 1942/43. Freigegeben von der FSK (Freiwillige Selbstkontrolle der Filmwirtschaft) ab 12 Jahre. Vorgeführt seit vier Wochen in vier großen Kinos der Stadt.

- „Anna“, ein Film von Franz Strecker mit der bliebten Jungschauspielerin Silvia Seidel, nach dem Roman „Anna Ballerina“ von Justus Pfaue. „Ein Ballettund Musikfilm um Liebe und Eifersucht“. Freigegeben von der FSK ab 16 Jahre. Läuft seit drei Wochen in acht Kinos der Stadt.

- „Vergessen Sie alles, was Sie wissen oder glauben zu wissen!“ „Willow“, ein Film von Ron Howard (Regie) und George Lucas (Produktion), den Schöpfern der "Star Wars" Trilogie und von "Cocoon“. Ein Phantasie-Film mit modernen mystischen Elementen über Angst und Geborgenheit, Bedrohung und Rettung, Gut und Böse. Freigegeben ab 12 Jahre. Läuft seit drei Wochen in fünf Kinos der Stadt.

- „Nummer 5 gibt nicht auf“ von Kenneth Johnson, ausgezeichnet mit dem Prädikat "besonders wertvoll“ der FBW (Filmbewertungsstelle Wiesbaden). Der Science-Fiction- und Abenteuerfilm quillt über vor Einfällen. Diesem Roboter-Burschen kann man nicht widerstehen. Freigegeben ab 6 Jahre. Läuft seit drei Wochen in vier Kinos der Stadt.

- „Das Dschungelbuch“, ein Klassiker von 1967. „Walt Disney's mit Abstand bester und unvergleichlicher Zeichentrickfilm. Freigegeben für alle Altersstufen. Lief als Leckerbissen in einem großen Kino der Stadt.

- "Cap und Capper". Freigegeben für alle Altersstufen. Läuft seit 5 Wochen in drei Kinos der Stadt, darunter in einem Drive-in-Autokino.

- Unbedingt erwähnen möchte ich auch noch den Film aus der Sowjetunion „Die Kommissarin“ von Alexander Askoldov. In der UdSSR nach 20 Jahren Aufführungsverbot freigegeben, in der DDR nach 25 Tagen im Kino verboten; seit neun Wochen im Kino, in München bereits über 140.000 Besucher. Freigegeben ab 12 Jahre. Läuft in drei Kinos der Stadt, die vorwiegend von Cineasten besucht werden.

Die Landeshauptstadt München hat auch drei Kinder-Kinos. Hier sind zu sehen: „Pan-Tau-Abenteuer in den Wolken“ (BRD/CSSR 1978), „Konrad aus der Konservenbüchse“ (BRD 1982), dem Film von Claudia Schröder nach einer Geschichte von Christiane Nöstlinger, „Die Schneekönigin“ (Finnland 1988) nach einer Erzählung von Hans Christian Andersen.

Eine Fülle von Filmen für Kinder und Jugendliche brachten über die Feiertage auch die deutschen öffentlich-rechtlichen und privaten Fernsehanstalten. Für die Zeit vom 17. bis 30.12 .1988 möchte ich auf einige markante Produktionen aufmerksam machen:

Das Erste Programm (ARD) zeigte:

- „Wenn der Wind weht“, englischer Zeichentrickfilm von 1985/86 über den Ernstfall des Atomkrieges. Jimmy Murakami gestaltete den Film nach dem Bilderbuch "Strahlende Zeiten" von Raymond Briggs. Das Entsetzten gewinnt durch die Zärtlichkeit und Naivität der Trickfiguren Jim und Hilda, einem Rentnerehepaar, eine bewegende Kraft. 
- „Andromeda, tödlicher Staub aus dem All“, amerikanischer Science-FictionFilm als futuristischer Alptraum, 1971 von Robert Wise, nach dem Roman „Andromeda“ des Wissenschaftlers Michael Crichton inszeniert. Tödliche Krankheitserreger aus dem All bedrohen die Erdenbürger.

Das Zweite Programm (ZDF) zeigte:

- „Der rote Strumpf“ von Wolfgang Tumler aus dem Jahre 1981 nach der Erzählung von Elfie Donelly. Es handelt sich um einen Spielfilm für Kinder, in dessen Mittelpunkt die Freundschaft zwischen einer 9jährigen und einer bereits älteren, einsamen Frau steht, die als „Sonderling" (Verrückte) abgestempelt wird.

- „Nonni und Manni“ (ZDF-Weihnachtsserie 1988), die Jungen von der Feuerinsel Island, von August Gudmundsson nach den Erzählungen von Jon Svenson verfilmt. In 6 Folgen wird das Schicksal der beiden Brüder erzählt. Schauplatz des Familienfilms ist das Island des 19. Jahrhunderts.

In den „Dritten Programmen“ (Regionalprogramme der ARD) wurden vorgeführt:

- „Pole Poppenspäler“ (Bayerischer Rundfunk, III. Programm/BR 3) nach der gleichnamigen Novelle von Theodor Storm (1872). In Rückblenden wird die Geschichte von dem friesischen Schüler Paul und der bayerischen Puppenspielertochter Lisei erzählt. Eine gemütvolle Literaturverfilmung für die ganze Familie.

- "Viktoria“ (BR 3), deutscher schwarz-weiß Spielfilm aus dem Jahr 1935 nach dem Roman von Knut Hamsun, in der Regie von Carl Hoffmann. Eine Liebesgeschichte und ein Sozialdrama um die Jahrhundertwende in Schweden.

Die privaten Fernsehstationen boten dem jungen Publikum folgende Produktionen an:

- „Doctor Dolittle“, USA 1966, von Richard Fleischer nach den Erzählungen von Hugh Lofting. Filmmusical mit verblüffenden Einfällen und phantasischen Abenteuern des Doctor Dolittle in der Begegnung mit Tieren und auf der Suche nach einer Riesenmeeresschnecke. (SAT 1)

- „Heidi und ihre Freunde“, deutsch-schweizerischer schwarz-weiß Spielfilm aus dem Jahr 1954/55 von Franz Schnyder nach der bekannten Erzählung von Johanna Spyri. Anspruchsloser, aber sympatischer Kinder- und Heimatfilm. (RTL-plus)

\section{Künstlerisch-formale und inhalts-orientierte Aspekte}

Thomas Schüly, einer der erfolgreichsten Filmproduzenten aus der Bundesrepublik Deutschland (Im Namen der Rose, 1986; Die Abenteuer des Baron Münchhausen, 1988), sagt über seine Arbeit: „Kino hat vor allem mit Entertainment zu tun. Ich will Leute für zwei Stunden auf einen Trip schicken. Ob das eine Komödie ist, ob das ein Drama ist, spielt überhaupt keine Rolle - die müssen mir auf dem Trip folgen ... Die müssen da rauskommen und tief in sich was gespürt haben, was ich noch bezeichnen würde als: wir sind Menschen. Und wenn Kino funktioniert, werden wir erschüttert!"11 
In ähnlicher Weise äußerte sich der bekannte Autor Thomas Mann, wenn er von der Literaturkaste etwas behauptet, das meiner Ansicht nach auch auf die Filmarbeit übertragbar ist: „Schriftstellertum ist Sensitivität und ein Voransein um mindestens fünf bis zehn Jahre“12.

\section{Was ist Literaturverfilmung?}

Verschwisterung zweier Künste oder ästhetisches Zwitterwesen? Die Verfilmung von Literatur läßt sich wohl als „interpretierende Transformation“ beschreiben. Die literarische Vorlage einerseits und das subjektive Werkverständnis des Filmemachers, sowie die Absichten die er mit seiner Produktion verfolgt andererseits, fließen in das cineastische Ergebnis ein. Der Betriff „filmische Literaturadaption" präzisiert, daß es sich um die Übertragung eines literarischen Werkes in das Medium Film handelt. Das Kinoprodukt steht dabei zur literarischen Vorlage in einem Verhältnis der Analogie. Daraus ergeben sich möglicherweise Diskrepanzen zwischen filmischer Realisation und den nicht minder subjektiven Erwartungen, sowie dem persönlich geprägten Vorverständnis der Zuschauer - oft Ursache für deren Unzufriedenheit mit der Verfilmung bereits bekannter literarischer Vorlagen. Der Autor Michael Ende war keineswegs zufrieden mit der Filmversion des erfolgreichen Kinohits „Die Unendliche Geschichte“ (Drehbuch: Hermann Weigel, Regie: Wolfgang Petersen). Die cineastische Verarbeitung seiner phantastischen Erzählung "Momo" hat Ende deshalb auch Rosemarie Fendel (Buch) und Johannes Schaaf (Regie) übertragen, mit deren Konzept er sehr einverstanden war.

Literaturverfilmung bewegt sich grundsätzlich zwischen zwei konträren Forderungen: einerseits sollte sie das schriftstellerische Werk in Gestalt, Thema und Idee möglichst adäquat wiedergeben, andererseits aber doch ein möglichst eigenständiges Filmkunstwerk abgeben. Die filmische Umsetzung von Literatur erfolgt hauptsächlich:

- auf „wörtliche“ Weise, als realistisches Ins-Bild-Setzen, quasi im Sinne von „Abbildhaftigkeit“. Damit wird jenen Literaturliebhabern Rechnung getragen, die eine unbedingte Werktreue, die buchstäbliche Bebilderung des dichterischen Wortes fordern;

- durch "Transformation" der literarischen Vorlage auf subjektive Weise. Dies bietet gewissermaßen eine mehr oder minder starke werkbezogene Paraphrasierung. Die Transformation spiegelt immer auch die persönliche Rezeption durch den Filmemacher wider.

Ich persönlich gebe diesem letzten Ansatz den Vorzug: Wortschöpfungen sollen meines Erachtens keineswegs nur vergangheitsbezogen und historizistisch oder in sklavischer Anpassung an die literarische Vorlage cineastisch verarbeitet werden. Ich ziehe es vor, wenn Literatur „bearbeitet“ wird, wie etwa bei einer zeitgenössisch-modernen Inszenierung eines klassischen Schauspiels oder einer alten Oper: heutigen Fragestellungen und heutigem Lebensgefühl gemäß aktualisiert. Auf diese Weise wird verbale Dichtung aus vergangenen Zeiten und aus anderen Kulturkreisen gegenwartsbezogen und mehrdimensional in dem Sinne, daß sie sich für politische, soziale, ethische und religiöse Ausdeutungen anbietet. 
Filmische Literaturadaptation bringt einige, immer wieder auftretende Probleme mit sich. Zwei Aspekte möchte ich ausdrücklich hervorheben: ${ }^{13}$

- Die Informationsfülle eines konventionellen Romans läßt sich nicht in der Laufzeit eines Kinofilms vermitteln. Deshalb wird der Stoff zumindest für das Fernsehen gelegentlich in mehrere Teile zerlegt. Als klassisches Beispiel hierfür kann die dreiteilige TV-Serie von Wolfgang Petersen „Das Boot“ (BRD 1979 bis 1981) gelten. Sie wurde von Kindern, vor allem aber auch von vielen Jugendlichen gern gesehen. Eine gekürzte Version der letzten Fahrt eines deutschen U-Bootes im Zweiten Weltkrieg, nach dem Erlebnisroman von Lothar Günther Buchheim aufbereitet, war vorher schon unter demselben Titel im Kino zu sehen - in gestraffter Form, unter Auslassung von Details und Episoden, von Randfiguren und Nebenhandlungen. Von dem schauspielerisch glänzend interpretierten Psychodrama der langen Fernsehversion, bleiben in der Kinofassung fast nur noch die martialischen Knalleffekte übrig und verkehren das antimilitaristische Heldentum der Vorlage Buchheims fast schon wieder ins Gegenteil.

- Die sprachlichen Zeichen des literarischen Werkes sind in den akustischvisuellen Code des Films umzusetzen. Weil der Film vorwiegend „situativ ganzheitlich" arbeitet, vermittelt er zunächst „gleichzeitig" verschiedene Eindrücke von äußeren Vorgängen: Sinneseindrücke, Bewegungsabläufe, Handlungen, Informationen aller Art, die in der Literatur über viele Seiten hinweg beschrieben werden müssen.

Das geschriebene Wort tut sich meist leichter als der Film bei der sukzessiven Darlegung innerer Vorgänge: Gedanken, Gefühle, Träume und Visionen. Sie sind mit den Mitteln des Tonfilms oft nur pantomimisch oder symbolischmetapherhaft zu bewältigen. Spezifische filmische Gestaltungsmittel wie etwa Einstellungsgröße, Kameraperspektive, Kamerabewegung, Zeitlupe und Zeitraffer, Überblendungseffekte usw. lösen beim Zuschauer andersgeartete Reaktionen aus als sie sich beim „Lesen“ einstellen.

Beide Zeichen-Systeme, beide Kommunikationsformen haben aber naturgemäß ihre jeweiligen Vorzüge und ihre spezifischen Grenzen, ihre Vor- und Nachteile. Der Filmbetrachter nimmt in erster Linie das vorgegebene Bild in seiner Totalität wahr, während der Leser die Bilder in seiner Phantasie allmählich entstehen läßt.

\section{Wie sollen Bücher und Filme für Kinder aussehen?}

Der Berliner Wissenschaftler Rudolf Müller stellte nach langen Untersuchungen mit 6 bis 12jährigen Schulkindern Kriterien für "gute" Kinderlektüre auf. Sie können auch für die Herstellung von Filmen für Heranwachsende gelten:

- Die Darstellungsweise soll aktionistisch, spannend, abenteuerlich sein nicht aber beschreibend, rein informativ. 
- Kinder haben noch einen ganz anderen „Humor“ als Erwachsene. Nicht den Wortwitz, das unerwartete „Umklappen“ einer Situation finden sie ulkig, sie brauchen keine Pointe, ja verstehen sie oft gar nicht. Kinder freuen sich über Szenen-Komik, an der spaßigen Darstellung von Personen (schmächtige Helden, große Ohren, roter Wuschelkopf). Sie sind begeistert über Streiche, die der Schwache dem Starken, die Kinder den Autoritätspersonen spielen.

- Die Handlung sollte phantastische Züge tragen und eher in einer dem Kind unbekannten Umwelt spielen als in der Alltagswelt. Speziell für Jugendbücher gilt: Neben Natur und Tieren sollten unbedingt auch Technik (Raumschiffe, Traktoren, Rennwagen, Hubschrauber) und Sport eine Rolle spielen.

- Ob für Buben oder Mädchen - wichtig ist noch: Körperliche Auseinandersetzungen in Kinderbüchern dürfen nie brutal sein. Aber es können durchaus Raufereien zwischen Kindern vorkommen. Soziale Probleme, Rollen- und Gesellschaftskritik sollen nicht plump eingetrichtert werden.

- Ellenlange Texte ohne Bilder schrecken ab. Doch bei zu vielen Bildern versteht das Kind die Handlung, ohne den Text lesen zu müssen. Besonders motivierend sind Buchreihen, weil die Kinder sich freuen, in den folgenden Bänden ihre Freunde wiederzufinden. Hier ist offensichtlich der gleiche Mechanismus anzutreffen, wie bei Fernsehserien.

\section{Literaturverfilmungen für Kinder}

Die Filmproduktion für Kinder und Jugendliche hat es nach wie vor schwer, sich durchzusetzen. In der kommerziell orientierten Kino- und Videoindustrie spielen Kinderfilme deutscher Herkunft eine nur marginale Rolle. Etwas besser sieht die Lage aus in den öfentlich-rechtlichen Fernsehanstalten. Die deutsche Filmgeschichte bietet dennoch eine ganze Reihe von Beispielen. Ich habe den Eindruck, daß man sogar von einer Renaissance an Literaturverfilmungen sprechen kann. Begründet wird diese Entwicklung unter anderem durch folgende Faktoren:

- Es gibt zeitgenössische Autoren und Autorinnen, deren schriftstellerische Werke offensichtlich aktuell genug sind oder die sich zumindest leicht verfilmen lassen. Dazu zähle ich etwa Heinrich Böll ("Das Brot der frühen Jahre“ 1961), Michael Ende (,die Unendliche Geschichte“ 1983, „Momo“ 1985) oder Christine Nöstlinger („Die Ilse ist weg“ 1976, „Konrad aus der Konservenbüchse“ 1982).

- Die Auszeichnung qualitativ hochwertiger Kinderbücher durch etwa 25 Kinder- und Jugendbuchpresse (Deutscher Jugendliteraturpreis, Katholischer Kinderbuch preis - fördert nicht nur die Lesekultur, sie macht auch Produzenten und Drehbuchautoren auf Stoffe für Kinderfilme aufmerksam. Die sehr beliebten Filme aus der Reihe "Janoschs Traumstunde“ (BRD 1980 ff.) - auch im Fernsehen als Serie gezeigt - sind nur im Zusammenhang mit den zahlreichen Janosch Kinderbilderbüchern zu sehen, die vorher schon hohe Aufla- 
gen erreicht und sich in die Herzen von Kindern (und junggebliebenen Erwachsenen) eingeschlichen hatten.

- Von der Pädagogik her wird die „Kunst des Erzählens“ und eine „Narrative Kultur“ ebenfalls wieder gefördert. „Erzählen“ hat erneut Eingang gefunden in unsere Kindergärten und Schulen. Ebenso zeitgemäß ist es, den Kindern daheim Geschichten vorzulesen oder Märchen zu erzählen. Ein weiteres Indiz dafür mag man auch in dem recht guten Verkauf von Hörkassetten mit zu Hörbildern gestalteten alten und neuen literarischen Stoffen sehen. Der AVVerlag „steyl-medien“ (München) hat davon in den letzten Jahren über 80.000 Exemplare abgesetzt.

\section{Filme in Kino, Fernseben und auf Video}

Die Zuordnung der nachfolgenden Filme zu thematischen Schwerpunkten dürfte im Einzelfall auch umstritten bleiben, sie orientiert sich dennoch an gemeinsamen, übergreifenden Kriterien.

\section{Wiederaufführung klassischer Literaturverfilmungen}

In München konnte man in den Kinos über Weihnachten und Neujahr 1988/89 zwei Klassiker nach Erzählungen von Erich Kästner sehen (geboren 1899), die jeweils mehrere Male verfilmt worden sind:

„Emil und die Detektive“, 1931 Regie: Gerhard Lambrecht, Drehbuch: Billy Wilder. Diese Urversion gilt als die erste Kinderbuchverfilmung in Deutschland überhaupt. Dabei stellte sich ein geradezu vorprogrammierter Erfolg ein, weil das Buch schon seit 1928 in breiten Schichten bekannt war! Was sich der Leser in der Geschichte in kühnsten Farben und Formen ausmalen kann, wird in dieser Verfilmung spannend interpretiert und mit filmisch einfachen, jedoch überzeugenden Mitteln sichtbar gemacht: Emil, der in Berlin seine Großmutter besuchen will, wird das mitzubringende Geld von einem Dieb entwendet; Emil verfolgt den Ganoven zunächst allein, wird aber bald von gleichaltrigen Freunden unterstützt, die den Verbrecher stellen ${ }^{14}$. Dieselbe Geschichte wurde 1954 eher glatt und vordergründig von R.A. Stemmle verfilmt. 1983 folgte schließlich noch eine US-amerikanische Walt-Disney-Version „Emile and the Detectives", eine etwas vergröberte, aber parodistische-muntere Kopie des klassischen Kinderromans, in der Peter Tewsbury Regie führte.

„Das fliegende Klassenzimmer" (BRD 1954), ein Schüler- und Jugendfilm, in der Regie von Kurt Hoffmann, unter intensiver Mitwirkung des Autors Kästner am Drehbuch. Die zweite, schwächere modernisierte Neuverfilmung erfolgte 1973 durch Werner Jacobs (Regie) unter Mitwirkung von Georg Laforet als Drehbuchautor. Immer wieder mit Erfolg aufgeführt wird auch der nach wie vor bezaubernde Zeichentrickfilm von Curt Linda "Die Konferenz der Tiere" aus dem Jahr 1969, ebenfalls nach einem Roman von Erich Kästner. 
Märchenwelle - Märchenfilme

Märchen als Spiegelungen menschlicher Grunderfahrungen sowie der herrschenden Sozial- und Wertordnung, wurden über Jahrhunderte hinweg mündlich tradiert. In Deutschland haben sie erst im 18. und 19. Jahrhundert ihre klassische schriftliche Form gefunden. „Die Märchen, die von den Brüdern Grimm gesammelt wurden, dienen als Beispiele dafür. Viele der Volksmärchen, die sie aufgezeichnet und auch geändert hatten, befassen sich mit Ausbeutung, Hunger und Ungerechtigkeit, welche die niedrigen Schichten in vorkapitalistischen Gesellschaften, besonders im Absolutismus, erlebt hatten. „Und der Zauber der Märchen - die wundervolle Hilfe und das heldenhafte Bestehen der phantastischen Prüfungen - können mit den Wunschvorstellungen und utopischen Projektierungen der bäuerischen Bevölkerung gleichgesetzt werden “15.

In Deutschland experimentierte man sehr früh mit der Bearbeitung von Märchen im Film: Paul Wegener produzierte die Stummfilme „Rübezahls Hochzeit" (1916) und "Der Rattenfänger von Hameln" (1919). Die wichtigste Vertreterin auf diesem Gebiet wurde jedoch Lotte Reiniger, die 1920 mit „Der verlorene Schatten" anfing, Scherenschnittfilme für Kinder zu machen (Silhouettenfilme, Schattentheater); „Die Geschichte des Prinzen Achmed“ aus dem Jahr 1926 - der auch als erster Animationsfilm der Filmgeschichte gilt - kann man sogar heute noch dann und wann sehen. Noch 1957 entstand der Reiniger-Scherenschnittfilm „Schneeweißchen und Rosenrot" mit einer Laufzeit von 12 Minuten.

Nach dem Zweiten Weltkrieg stritten sich die Märchenfilm-Produzenten und -Verleiher Schonger, Genschow, Fürster, Delos, Zengerling und Diehl um die Adaptation, so daß manche Märchen gleich mehrere Male verfilmt worden sind, vor allem in den 50er Jahren. Dann verebbte der Enthusiasmus im Zuge einer aufklärerischen Erziehung bis in unsere Gegenwart. Erst in den letzten Jahren haben Märchenfilme in der Bundesrepublik eine Wiederbelebung erfahren. Aufhorchen ließ der Psychoanalytiker Bruno Bettelheim mit seiner 1977 bei uns erschienenen Schrift „Kinder brauchen Märchen"16. In Zusammenarbeit mit der in München erscheinenden „Kinder- und Jugendfilmkorrespondenz" wird seit 1983 ein „Projekt zur Wiederentdeckung der Silhouettenfilme von Lotte Reiniger" durchgeführt. Das „Kinder- und Jugendfilmzentrum in der Bundesrepublik Deutschland“ hielt 1986 eine Fachtagung ab zum Thema "Märchen im Film" unter dem Motto „Vom Zauberwald zur Traumfabrik"17.

Neue Verfilmungen der klassischen Märchen entstanden, zum Beispiel auch in Koproduktion mit der Tschechoslowakei. Ich erinnere nur an die recht freie Verfilmung der Grimmschen Vorlage „Frau Holle“ (Perinbaba) aus dem Jahr 1984 durch Juraj Jakubisko (Regie) und Lubomir Feldek (Buch): ein insgesamt phantasie- und stimmungsvolles Abenteuer für Kinder und Erwachsene. Aus der DDR wurde der 1987 entstandene Puppentrickfilm „Zwerg Nase“ (Regie: Katja Georgi; Buch: Katja Georgi/Eberhard Görner) nach dem Märchen von Wilhelm Hauff übernommen: Der kleine Jakob, durch Zauberei zum Koch mit der langen Nase geworden, kehrt am Ende als normaler Junge wieder zu seiner Mutter zurück. Das Institut für Film und Bild (FWU) in Grünwald bei München brachte 1987 nach Ludwig Bechsteins Vorlage "Das Nußzweiglein" den Legetrickfilm "Das Mädchen und die Bestie" heraus (Regie: Michael Schaak). 
Nicht unerwähnt bleiben dürfen in dem Zusammenhang die vom USAmerikaner Terry Gilliam erneut verfilmten „Abenteuer des. Baron Münchhausen“ nach den Erzählungen gleichen Namens: „Da ist ein alter Mann, der nicht mehr leben will, dem keiner glaubt; und der begegnet einem Mädchen, das ihm hilft, ihm vertraut und ihn manchmal wie eine Mutter beschützt, ihn zurück ins Leben bringt. Und der Baron, dem man plötzlich seine Geschichten glaubt, die dann auch Realität werden, wurde von uns entwickelt, ohne daß wir uns sehr an die Vorlage gehalten hätten. Der Ur-Münchhausen, der übrigens zum ersten Mal in Englisch in London erschienen ist, umfaßt ohnehin nur 42 Seiten $^{\text {"18 }}$. Ein 40 Millionen-Dollar-Spaß für Jung und Alt.

\section{Moderne Märchen: Science-Fiction- und Phantasie-Filme}

Die „neuen Helden“ sind neugierig, freundlich, klug und gerissen, frech und verträumt, manchmal ängstlich, meist mutig und trotz ihrer Streiche, des Widerspruchs, der Dickköpfigkeit und Selbständigkeit der Stolz ihrer Eltern und Nachbarn. Sie begegnen mitten im oft ereignislosen und eher langweiligen Alltag ganz zufällig Ganoven und Gangstern, Magiern und Geistern, allerlei skurilen Außerirdischen; sie finden Schätze, fliegen mit Ufos, haben Ärger mit Monstern oder telefonieren ganz selbstverständlich mit dem verunglückten Vater oder der verstorbenen Schwester. Ihre Reaktionen auf das Ungewöhnliche, Phantastische, Mysteriöse sind ebenfalls ungewöhnlich. Doch am Ende des Films ist die Welt wieder in Ordnung, die Kinder sind etwas reifer und die Erwachsenen ein wenig verständnisvoller geworden.

Erstmals aufgetaucht sind diese „neuen Helden“, wenn ich richtig sehe, in Steven Spielbergs „Unheimliche Begegnung der Dritten Art“ (USA, 1977). Anders als die genreüblichen "Invasionsfilme“, entwirft Spielberg die Utopie einer von Harmonie und Freundlichkeit erfüllten außerirdischen Welt, die den Menschen "guten Willens" und „reinen Herzens" offensteht. Dramaturgisch und technisch ein mit bemerkenswerter visueller Phantasie und handwerklicher Raffinesse gestalteter Märchenfilm. 1981 führte Spielberg diesen Ansatz mit „E.T. - Der Außerirdische“ zur Perfektion.

In Roland Emmerich's ,Joey“ (BRD 1985) nutzt ein 9jähriger Junge seine telekinetischen Fähigkeiten zur Kontaktaufnahme mit seinem verstorbenen $\mathrm{Va}$ ter. Dabei werden die freigesetzten übersinnlichen Fähigkeiten von bösen Kräften bedroht - was zu einer Kette von Katastrophen führt. „Enemy Mine - Geliebter Feind" (BRD/USA, 1985), nach einer Novelle von Barry Longyear erzählt wie ein irdischer Kampfpilot und ein außerirdisches Wesen mit gefährlichen Ereignissen auf einem unerforschten Planeten konfrontiert werden. Nach anfänglichem Mißtrauen, werden sie zu Freunden. Zu dieser Sorte würde ich auch die nach dem gleichnamigen Roman von Michael Ende verfilmte „Unendliche Geschichte" rechnen (BRD, 1983), eine überaus aufwendige Verfilmung unter der Regie von Wolfgang Petersen: Ein Junge liest von Abenteuern im Land Phantasien, das vom unfaßbaren „Nichts“ bedroht wird und er erkennt dabei, daß er selbst Teil dieser Geschichte ist. Mit Engagement und Phantasie, und mit einer Reihe skuriler Helfer, kann er schließlich überwinden, was die Hoffnungslosigkeit der Menschen nährt. Dazu zählt schließlich auch die Verfilmung des 
gleichnamigen Erfolgsromans von Michael Ende „Momo“ (BRD 1986) in der Regie von Johannes Schaaf: Eine liebenswerte Menschengruppe wird von bösen „Zeitdieben“ bedroht, jedoch von einem kleinen Mädchen im Bunde mit dem gottähnlichen „Meister Hora" gerettet. Die Inszenierung ist weniger durch technische Effekte und eher durch glaubhafte Charaktere und athmosphärische Dichte gekennzeichnet, wenngleich manche Passagen eher auch etwas betulich wirken.

\section{Familie und Schule, Freundschaft und Liebe}

Hier handelt es sich um wichtige Lebensräume, aber ebenso um emotionale, soziale und geistige Werte, die für Kinder und Jugendliche von existentieller Bedeutung sind. Nach spezifischen Jugendfilmen wird in der Bundesrepublik Deutschland händeringend gesucht. Familienfilme haben sich dagegen inzwischen als „Familienserien“ einen festen Platz im deutschen Fernsehen erobert.

Nach dem gleichnamigen Roman von Christine Nöstlinger verfilmte der „Kinderfilmer" Hark Bohm 1975 „Wir pfeifen auf den Gurkenkönig“. In zweifacher Hinsicht ist dieser Film von Bedeutung: Bohm hat damit den Aufschwung des "neuen deutschen Kinderfilms" mitgeprägt, der Mitte der 70er Jahre einsetzte; und erstmals hat das Deutsche Fernsehen (SR-Saarbrücken) Mittel aus dem Unterhaltungsprogramm abgezogen und mit einer aufwendigen Eigenproduktion (DM 700.000,--) an die hervorragenden Erfahrungen angeknüpft, die man vorher mit übernommenen Spielfilmen gemacht hat: „Christine Nöstlinger versagt sich bei dieser Parabel auf autoritäre Strukturen in der Familie jeglichen 'Märchenton'. Sie erzählt manchmal derb-realistisch, wobei es ihr darauf ankommt, daß3 Kinder sich in der Situation wieder erkennen. Ebenso verfährt Hark Bohm im Film mit Dialogen, die teilweise noch akzentuierter sind als im Buch $^{\text {“19. }}$. Bohm führt Kindern ihre tägliche Lebenssituation in Familie und Schule vor Augen, zeigt ihnen die Macht und die Gewalt, der sie ausgesetzt sind; beweist ihnen Solidarität als Möglichkeit, ihnen zu begegnen.

Neueren Datums ist die Verfilmung eines weiteren Romans von Christine Nöstlinger „Die Ilse ist weg“ (BRD 1976) durch Ilse Hoffmann: An der schönen Ordnung des gutbürgerlichen Familienlebens ist etwas faul; so sehr, daß Ilse ausbricht. Hinter der heiter-ernsten Geschichte werden Erziehungsprobleme sichtbar. Neu ist Marianne Rosenbaums Verfilmung des erfolgreichen Kinderbuchs von Gudrun Mebs „Sonntagskind“ (BRD 1988); „Während das Buch aus der Sicht des Kindes geschrieben ist, das im Heim lebt und darauf wartet, einmal in der Woche von der 'Sonntagsmami' abgeholt zu werden, erzählt Marianne Rosenbaum die Geschichte der Frau und des Kindes: Eine Schriftstellerin holt sich zu Recherchenzwecken ein Kind aus dem Heim. Aus dem Recherchenobjekt entsteht ein Subjekt, das von ihr geliebt wird und liebt. $\mathrm{Zu}$ den inneren Konflikten kommen äußere; da ist zum Beispiel die Schwierigkeit einer nichtverheirateten, freiberuflichen Frau, ein Kind zu haben, erst recht eins zu adoptieren." Die Autorin Gudrun Mebs war mit den Akzentsetzungen der Regisseurin durchaus einverstanden und hat selbst die Rolle der Mutter im Film übernommen. ${ }^{19}$ 
Abenteuer - Krimis - Reiseberichte

Hunger der Kinder nach „Action" und "Erleben", nach starken Gefühlen von Bedrohung und Zärtlichkeit, nach Ausbruch aus der oft monotonen oder gar tristen Alltagswelt, nach Spannung - mit den Medien und jenseits derselben - ist schier grenzenlos. Abenteuer- und Kriminalfilme sowie Reiseberichte über fremde Länder und Völker, ihre Sitten und Gebräuche kommen diesem Bedürfnis entgegen.

Beliebt sind Robinson-Geschichten nach den Erzählungen Daniel Defoe's. Von deutscher Seite steuerte der Regisseur Josef von Baky bereits 1956 nach dem gleichnamigen Theaterstück und Jugendbuch von Friedrich Forster den warmherzigen und frisch inszenierten Unterhaltungsfilm „Robinson soll nicht sterben" bei. Frei nach einem Roman von Karl May wurde von Regisseur Harald Reinl 1968 „Winnetou und Old Shatterhand im Tal der Toten" verfilmt - nur eine erfolgreiche Kinoproduktion dieser Art von vielen.

Bei vielen Heranwachsenden sind die humorvoll-spannenden Unternehmungen der Amateur-Detektivin Miss Marple nach den Kriminalgeschichten von Agatha Christie (1890 bis 1976) nach wie vor gern gesehen! Diese typisch britischen Kriminal-Kommödien mit ihrem trockenen Humor wurden alle auch deutsch synchronisiert. Sie verdanken ihren Erfolg in Kino und Fernsehen vor allem der liebenswürdigen Komik der Hauptdarstellerin Margareth Rutherford, mit ihrer massigen Körperfülle und durch nichts zu erschütternden Gelassenheit. Petra Haffters neuer Film „Der Kuß des Tigers“ (BRD/F 1988) nach der Kriminalgeschichte "Wollen Sie mit mir sterben?" von Francis Ryck (rororo, Frankfurt am Main) ist ein Thriller über eine gefährliche Leidenschaft. Auf eine harte Probe gestellt wird die Liebe zwischen Petra, einem lebenslustigen Aupair-Mädchen in Paris und Peter, einem Frauenmörder auf der Flucht vor der Polizei und auf der Suche nach sich selbst.

\section{Musik- und Tanzfilme}

1967 kämpften die Beatles in dem Realspiel- und Zeichentrickfilm „Yellow Submarine“ (GB 1987, Regie: George Dunning) mit ihren Songs gegen die Mächte der Eintönigkeit, des Amusischen und Unmenschlichen. Der Film „Saturday Night Fever" (USA 1978) mit Olivia Pascal und John Travolta als Hauptdarsteller löste 1978 einen neuen Tanz-Kult bei Jugendlichen aus, die sich seitdem am liebsten in Diskotheken vergnügen. Seit dem Kinostart von „Dirty Dancing" (USA 1987) melden die Tanzschulen vermehrte Nachfrage nach MamboKursen. Musik- und Tanzfilme kommen dem Bewegungs-, dem Partnerschaftsund Kommunikationsbedürfnis Jugendlicher entgegen. Gerade in Lieder und Rhythmen drücken sie gerne ihr Lebensgefühl aus.

Gloria Behrens hat in ihrem 1980 fertiggestellten Film „Rosi und die große Stadt" die gegenwarts- und problembezogene Story des kleinen Mädchens aus einem bayerischen Dorf, das mit Vater und Bruder nach Berlin verschlagen wird, als schmissiges Rock-Musical inszeniert. Musik und Tanz wurden dabei geschickt in die Realhandlung einbezogen. Neueren Datums, und für Jugendliche und Erwachsene vorgesehen, ist Reinhards Hauffs Verfilmung des erfolgrei- 
chen Musicals des Berliner Grips-Theaters „Linie 1“ (BRD 1987, Text: Volker Ludwig, Musik: Birger Heymann), es erzählt die Geschichte des Mädchens Sunnie, das auf der Suche nach einem Rocksänger aus der Provinz in die Großstadt kommt.

Die Fernsehserie „Anna“ (BRD 1987) - als Weihnachtsserie des ZDF bereits Ende 1987 ausgestrahlt - wurde durch die begabte junge Schauspielerin Silvia Seidel populär, weshalb wohl auch ein Buch zur Serie herausgekommen ist - wieder einmal der umgekehrte Weg, was ja immer häufiger geschieht. Regisseur Frank Strecker wollte mit erheblichem technischen Aufwand den Rahmen der üblichen TV-Produktion sprengen, behielt aber bei der Herstellung des Kinofilms den Stil netter Jugendsendungen und Familienfilme bei: Adretter Teanager zwischen Tanzvergnügen und Balettkarriere, zwischen Eifersucht und Liebe $^{20}$.

\section{Geschichte und Vergangenheitsbewältigung}

Der in Augsburg aufgewachsene Schriftsteller Berthold Brecht hat einen Satz formuliert, der gut zu diesem Abschnitt paßt: „Die Zukunft wird abhängen von der Erledigung der Vergangenheit". Mit "erledigen" ist hier allerdings nicht das "Vergessen" gemeint, sondern vielmehr das Wahrhabenwollen und Bewußtwerden von Vergangenem; und vor allem das Bekenntnis zu wertvollen Traditionen aus der Geschichte sowie die Aufarbeitung und Bewältigung von jüngster Vergangenheit. Nach dem Bühnenstück von Berthold Brecht, drehten Peter Palitzsch und Manfred Wekwerth den gleichnamigen Film „Mutter Courage und ihre Kinder" (BRD/DDR 1960). Das Schicksal der Marketenderin, die im 30jährigen Krieg ihren Profit zu schlagen versucht, dadurch aber ein Kind nach dem anderen verliert, ist nach der unter Brecht selbst erfolgten Modellinszenierung des „Berliner Ensembles“ verfilmt.

Über das Aufkommen des Faschismus in Deutschland in den Jahren 1932/33, über Arbeitslosigkeit und Diskriminierung von Andersdenkenden, über Freundschaft und Kinderbanden berichtet der Film „Die Kinder aus Nr. 67" (BRD 1979/80). Er wurde nach der gleichnamigen Romanreihe von Lisa Tetzner von Usch Barthelmeß-Weller und Werner Mayer gedreht. Der sorgfältig inszenierte und mit einer bemerkenswert zeitgetreuen Ausstattung versehene Film schildert die Zustände Anfang der 30er Jahre aus Erleben und Sicht einer Clique von Kindern. Leonie Ossowski schrieb das Drehbuch selbst, nach ihrem gleichnamigen Roman. Ottokar Runze frühte Regie bei „Stern ohne Himmel“ (BRD 1980): Kurz vor Ende des Zweiten Weltkrieges entdecken 15jährigen Schüler einen aus dem $\mathrm{KZ}$ geflohenen jüdischen Jungen. Sollen sie ihm helfen oder müssen sie ihn verraten? Aus der Sicht seiner jugendlichen Helden hält der Film dem Haß und der Unmenschlichkeit in chaotischer Zeit ein Beispiel praktizierter Nächstenliebe entgegen.

Percy Adlons Spielfilm „Fünf letzte Tage“ (BRD, 1982) schildert die letzten Tage im Leben der jungen Widerstandskämpferin Sophie Scholl, erzählt aus der Sicht ihrer Mitgefangenen Else Gebel. Grundlage für die Arbeit des Filmautors waren, neben Dokumenten und Interviews mit Überlebenden, die Auf- 
zeichnungen von Sophies Zellengenossin Else. Gerade durch die Aussparung unmittelbarer Grausamkeiten, wird der banal-alltägliche Schrecken nationalsozialistischer Machtausübung erlebbar. Ebenfalls 1982 erschien noch Michael Verhoevens Dokumentarspiel „Die weiße Rose“. Er zeichnet die Geschichte dieser studentischen Widerstandsgruppe um die Geschwister Sophie und Hans Scholl nach, die in München gegen das Terrorregime des Nationalsozialismus kämpfen, bis sie 1943 von der Gestapo (Geheime Staatspolizei) aufgespürt und hingerichtet wurden. Das Drehbuch stützt sich ebenfalls auf Dokumente, Aufzeichnungen und Gespräche - die ihren Niederschlag auch anderswo in Publikationen gefunden haben.

\section{Aktuelle Fragen und zeitkritische Themen}

In Film und Literatur widerspiegeln sich Verhältnisse der Gesellschaft: Medien sind Ausdruck und Deuter der Zeit! Bestimmte Themen bewegen die Öffentlichkeit und beeinflussen damit auch Denken, Fühlen und Verhalten von Kindern und Jugendlichen. Der Spielfilm von Gerhard Widmer "Was heißt hier nach Hause“" (BRD 1986) setzt mit geringfügigen Abweichungen und Aktualisierungen den Jugendroman aus dem Jahr 1978 von Sigrid Schuster-Schmah um „Staatsangehörigkeit: Griechisch“. Er behandelt die Wiedereingliederung "emanzipierter" Gastarbeiterkinder der zweiten und dritten Generation in eine patriarchalisch strukturierte Gesellschaft. Die Geschichte des Mädchen Irmia und ihres älteren Bruders bietet Anknüpfungspunkte für Ängste, Erwartungen und Hoffnungen. Ausgehend von der gleichnamigen Erzählung von M.J.M. Wippersberg „Fluchtversuch“ (A/BRD 1976) verfilmte Vojtech Jasny bereits 1976 die Geschichte eines Gastarbeiterkindes, das sich in Wien zahlreichen Vorurteilen ausgesetzt sieht und sich deshalb auf den Weg heim nach Jugoslawien macht. Viele Probleme, die sich für Kinder aus Fremdarbeiterfamilien wie auch für deutsche Kinder ergeben, lassen sich anhand des Films gut behandeln. Mit dem Prädikat „besonders wertvoll“ ausgezeichnet wurde der Dokumentarspielfilm „Aus der Ferne sehe ich dieses Land“ (BRD 1978) von Christian Ziewer nach dem Jugendbuch von Antonio Skarmenta. „Nix passiert“: Der 16jährige Lucho hat mit seiner Familie aus Chile fliehen müssen, nachdem die Militärjunta unter General Pinochet die Anhänger Allendes verfolgt und das demokratische System abgeschafft hatte. Aber das Leben im Berliner Exil ist keineswegs leicht.

„Kraftprobe“ (BRD 1982) wurde von Heidi Genée nach dem Roman „Ich bin eine Wolke“ von Dagmar Kekule gedreht: Kirstin, eine 15jährige muß sich daheim allein durchschlagen, weil die Mutter in einer Entziehungsanstalt weilt. Auch in diesem Fall kam es zunächst zu einem Konflikt zwischen dem Produzenten und der Buchautorin: „Die Telefilm Saar hatte zunächst vor, den Film mit Erwin Keusch (Schweiz) zu machen, aber der wollte eher eine 'Teenager-LoveStory', womit Dagmar Kekule nicht einverstanden war. Sie wurden sich auch nicht einig. Ich bin dann dazu gekommen, wollte das Buch zuerst gar nicht lesen, weil ich mir nicht vorstellen konnte, wie das funktionieren soll, wenn ich das Drehbuch nicht selber geschrieben habe. Während des Lesens habe ich mich aber so begeistert, daß ich dachte: 'Gut, wenn mir so was schon nicht selbst eingefallen ist, diese Mischung aus Kraft, Humor und Sozialstudie, dann will ich es wenigstens realisieren ${ }^{21}$. 
Nach Jürgen Breest's Buchvorlage "Tollwut" gestaltete Ilse Hofmann den gleichnamigen Kinderfilm (BRD 1981): Er handelt von den Schwierigkeiten zweier unterschiedlicher Familien und den daraus entstehenden Konflikten zwischen den Jungen. Mit großem Erfolg liefen in der Bundesrepublik die mehrfach ausgezeichneten Filme „Zwei Welten - A World Apart“ (GB 1988) von Chris Menges und "Schrei nach Freiheit" (Cry Freedom, GB 1987) von Richard Attenborough. Menges Geschichte geht zurück auf einen authentischen Fall und beschreibt nach dem autobiographischen Stoff von Shawn Slovo, wie ein 13jähriges Mädchen in den 60er Jahren das Unterdrückungsregime in Südafrika zu durchschauen beginnt. Attenboroughs Film basiert auf den autobiographischen Aufzeichnungen des südafrikanischen Journalisten und Zeitungsverlegers Ronald Woods über seine Begegnung mit dem Bantu-Führer Steven Biko, der 1977 im Gefängnis an Mißhandlungen starb. Hans Henning Borgelt verfilmte nach dem gleichnamigen Kinderbuch von Elfi Donelly „Servus, Opa, sagte ich leise" (BRD 1979). Michi, 10 Jahre alt, wird durch seinen Opa, der im Sterben liegt, mit Alter, Krankheit und Tod konfrontiert. Aber auch mit der Unbeholfenheit und Unsicherheit, ja sogar mit dem oft heuchlerischen Verhalten der Erwachsenen.

Dieser Thematik nahm sich jüngst auch Haro Senft an mit der Verfilmung der Erzählung gleichen Namens von Peter Härtling „Jakob hinter der blauen Tür“ (BRD 1978). Ein Film über Tod und Trauern in unserer heutigen Gesellschaft. Senft über die Umsetzung der geschriebenen Geschichte in ein filmorientiertes Drehbuch: „Von Anfang an war klar, daß man natürlich die Version von Peter Härtling nicht so direkt verfilmen konnte, denn es hätte eines großen Geld- und Zeitaufwandes bedurft, um die Phantasien und Projektionen des Jungen wirklich gut und ausführlich zu zeigen. Wir waren also bemüht, eine Form zu finden, die sich mehr auf dem realen Vorfall und der realen Ebene bewegt und gleichzeitig auch eine sehr zeitnahe Interpretation ist. Das Thema selbst ist zeitlos, aber es sollte doch so zeitentfernt nicht sein, wie es bei Peter Härtling vorkommt. Ich habe kurz vor Drehbeginn noch einmal mit Härtling gesprochen und gefragt, was denn das signifikante an seinen Figuren sei, und er sagte, bei der Mutter vorwiegend die Unfähigkeit zu trauern, bei dem Jungen die Bemühung, damit fertig zu werden ${ }^{22}$.

Religiöse und methaphysische Themen

Sowohl die Bibel wie auch die Geschichte der Kirche haben nicht nur Schriftsteller inspiriert. Immer wieder haben sich auch Drehbuchautoren und Regisseure große Themen der Religion und markanter Ereignisse christlicher Kulturgeschichte angenommen - bis in unsere Tage! Es gibt nicht nur eine eigenständige, breite religiös-orientierte Kinderliteratur, vielmehr sind auch eine ganze Reihe von Filmen für Heranwachsende zu verzeichnen, die religiöses Brauchtum und kirchliches Leben, christliche Grundwerte und Spiritualität zum Gegenstand haben.

Nach einem archäologisch-orientierten Roman von Werner Keller verfilmte Harald Reinl im Stil eines Kulturfilms ,... und die Bibel hat doch recht" (BRD 1977). Er versucht optisch zu zeigen, daß archäologische Funde und 
Erkenntnisse der Geschichtswissenschaften nicht im Widerspruch zu biblischen Aussagen stehen. „Hiobs Revolte“ (BRD/Ungarn 1982), nach autobiographischen Aufzeichnungen von Imre Güyöngyössy, Barna Kabay und Katalin Peteniy filmisch gestaltet, schildert aus der Sicht eines etwa 7jährigen, wie ein jüdisch-chassidisches Bauernehepaar in Ungarn einen weißen Jungen adoptiert, den es als Erben seines Glaubens und seiner Kultur vor der Verfolgung durch den Faschismus bewahrt. „Wir sind Utopia“, nach der gleichnamigen Novelle von Stefan Andres, von Dagmar Dameg gedreht (BRD 1986), erzählt die Geschichte des Matrosen Baco. Während des spanischen Bürgerkrieges wird er, zusammen mit anderen Kriegsgefangenen, in ein altes Karmeliterkloster eingeliefert, das zum Gefängnis umfunktioniert worden ist. Für Baco ist der Ort nicht unbekannt: Vor 20 Jahren lebte er hier als Padre Consalves, bis er aus dem Orden austrat und das Kloster verließ. "Flammenzeichen“ (BRD 1984) von Franz Seitz zeichnet das Leben des Jesuiten Ruppert Mayer nach, der als Seelsorger in München unbeugsam der Ideologie des Nationalsozialismus trotzte. Das Drehbuch von Gabriele Seitz stützt sich teils auf das Theaterstück ${ }_{n}$ Pater Ruppert Mayer S.J." von Walter Rupp, teils auf Dokumentarmaterial.

Mit „Sei Still, wisse: Ich bin“ (BRD 1982) vertonte der Komponist Florian Fricke Texte aus den 1947 in einer Höhle gefundenen Schriftrollen von Qumran für Chor und Instrumentalbegleitung in gehobenem Pop-Stil. Filmisch inszenierte er dann das Oratorium in der Landschaft am Toten Meer und in der Wüste Sinaj. Der Experimentalfilm stellt hohe Anforderungen an das Publikum, wird von Jugendlichen wegen der meditativen Texte und Melodien aber gern gesehen.

Für die Kleinen, schon ab etwa 6 Jahren, wäre die Verfilmung verschiede-

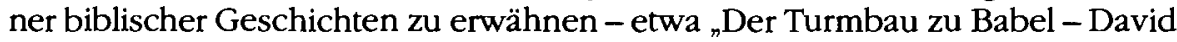
und Goliath - Josef und seine Brüder - Jona" (alle von Tellux-Film GmbH, München). Ebenso für Kinder ab etwa 6 Jahren gedacht ist die Verfilmung der Gleichnisse „Der reiche Mann - Der verlorene Sohn - Das Urteil" von Jaro Ucen (Studio R, Kirchheim bei München).

Für Jugendliche ab etwa 12 Jahre ist in den letzten Jahren im Institut für Film und Bild, München (FWU) ebenfalls eine Filmreihe über Gleichnisse Jesu herausgekommen: Nicht vordergründig-altertümlich gestaltet, sondern in unsere Zeit verlegt und für die Gegenwart ausgelegt: „Im Ernstfall“ (1977) visualisiert das Gleichnis vom reichen Kornbauern (LK 12,16-20); „Rückkehr“ (1980) thematisiert das biblische Gleichnis vom verlorenen Sohn, der im Film aber eine Tochter ist (LK 15,11-32). Im Hintergrund des Kurzspielfilms „Das Wagnis“ (1981) stehen die Doppelgleichnisse vom verlorenen Schaf und der verlorenen Drachme (LK 15,1-10), sowie vom Schatz im Acker und der kostbaren Perle (Mt 13,44-46). Der gegenwartsnah inszenierte Kurzspielfilm „Das Gastmahl“ (1981) visualisiert das Gleichnis vom königlichen Hochzeitsmahl (Mt 22,14) bzw. vom Festmahl eines Reichen (LK, 14,15-24). „Die Vereinbarung“ (1982) aktualisiert die biblische Erzählung von den Arbeitern in Weinberg (Mt 20,1-16). „Daniel. Menschen im Widerstand“ (1986), ist ein Legetrickfilm über die ersten Kapitel des Buches Daniel, verbunden mit zeitgemäßen Fragestellungen und heutigem Lebensgefühl. 
Ich vermisse in der Bundesrepublik Deutschland eine ${ }_{n}$ Kultur des Kurzfilms“. Seitdem diese Filmgattung - aus vielerlei Gründen - Mitte der 60er Jahre aus den gewerblichen Filmtheatern nahezu gänzlich verschwunden ist, hat sich kein Ersatz eingestellt. Das Fernsehen bot dafür keinen angemessenen Ausgleich. Wie Glosse, Kurzgeschichte oder Novelle in der Literatur, so müßten meines Erachtens auch Kurzfilme als cineastische Kunstwerke, als Ausdruck und Deuter der Zeit ihren festen Platz in der Kulturarbeit haben.

„Langfilme haben ihre Berechtigung, sie können große Geschichten erzählen, geben Zeit zum Einfühlen, schaffen Filmerleben, haben intensive Wirkung. Insbesondere für kleinere Kinder werden sie jedoch nicht selten zur Belastung, denn langes Stillsitzen ist eben wenig kindgerecht. Daher erscheint es sinnvoll und notwendig den kurzen Film für Kinder kinofähig zu machen. Kurzfilme also nicht nur in den internen Bereichen wie Kindergarten und Schule, sondern auch im öffentlichen Bereich des Kinderkinos einzusetzen. Als fester Bestandteil im Kinderkino hat der Kurzfilm nur dann eine Chance, wenn er den Gewohnheiten und Erwartungshaltungen der Besucher sowie den organisatorischen und ökonomischen Zwängen (z.B. Eintrittsgelder) eines (Kinder-)Kinos entgegenkommt, das heißt eine bestimmte Mindestdauer nicht unterschreitet. Etwa 40 Minuten Filmprogramm als zeitliche Untergrenze hat sich in verschiedenen nichtgewerblichen Kinderkinoeinrichtungen bewähr ${ }^{23}$.

Zahlreiche Literaturverfilmungen sind gerade auch aus dem Kurzfilmbereich zu verzeichnen. Stichwortartig erwähne ich nur:

- Verfilmung klassischer Volksmärchen, etwa „Der Tannenbaum“ nach Hans Christian Andersen;

- Zeitgenössische Erzählungen, etwa die Trickfilme „Oh wie schön ist Panama“ oder „Komm, wir finden einen Schatz" von Janosch;

- Phantastische Geschichten, etwa „Als die Igel größer wurden“: ein Trickfilm, der eine Geschichte aus dem Jahre 2180 erzählt, die Wirklichkeit werden könnte;

- Aktuelle Themen etwa: „Computerzeit", die Welt der Elektronenrechner, für Kinder erklärt. Oder auch „Mutmachergeschichten“ und „Bettkantengeschichten", die als generationsverbindende Erzählungen in Szenen gesetzt werden.

\section{Schlußanmerkungen}

Bei der Situationsbeschreibung fällt auf: Die Zusammenarbeit zwischen Schriftstellern einerseits und Filmemachern andererseits funktioniert im Vorfeld der Produktion offensichtlich erstaunlich gut. Etliche Autoren haben selbst Drehbücher geschrieben, viele waren zumindest beratend und auch begutachtend an der Erstellung der Drehbücher beteiligt. In wenigen Fällen, wie beispielsweise in "Sonntagskind" von Marianne Rosenbaum, haben sie sogar im 
Film mitgewirkt. Nur in seltenen Fällen kam es zwischen Schriftstellern und Filmproduzenten zu Unstimmigkeiten, wie etwa bei der „Unendlichen Geschichte" zwischen Michael Ende und Wolfgang Petersen. Erstaunlich ist auch, daß - vor allem bei neueren Literaturverfilmungen - viele Autorinnen mit ihren Werken vertreten sind: Christine Nöstlinger, Elfie Donelly, Gudrun Mebs, Dagmar Damek, Dagmar Kekule, Leonie Ossowski, usw. Ihre Beiträge beziehen sich eher auf aktuelle Alltagsprobleme sowie auf die Darstellung menschlicher Beziehungen; und weniger auf Abenteuer, Krimis oder phantastische Geschichten.

Beide Kunstgattungen - Buch und Film - sind anregend und aufregend, beschwichtigend und befriedigend. Denn Bücher wie Filme sind ein getreuer Spiegel unserer hektischen Welt, der Träume und Alpträume des Menschen. Sie sind Ausdruck unserer Existenz ein Portrait der Zeit. Sie nehmen vorweg, was heute von jedem der sehen kann, zu sehen ist: all das in uns Erschütterte.

Beziehungen zwischen Literatur und Film gibt es. Und es sieht sogar so aus, als käme es immer mehr zu einem gegenseitigen Austausch. Wir erleben nicht nur eine Konkurrenz der Medien, sondern zunehmend auch ihre wechselseitige Durchdringung und Verzahnung.

\section{Anmerkungen:}

$1 \mathrm{Vgl}$. Ulrich Beer: Umgang mit Massenmedien, Tübingen 1968

2 Vgl. Marie Winn: Die Groge im Wohnzimmer, Rowohlt Verlag, Reinbek bei Hamburg 1979.

3 Albrecht Weber: „Literatur: Lesen, Hören und Sehen?" in: Mitteilungen des Germanistenbundes, Frankfurt/M. 1981, Seite 9.

4 Marin Keilhacker, in: Filmpädagogen 1917, zitiert nach „Rheinpfalz“, Ludwigshafen 24.11.1956.

5 Zitiert nach „Westdeutsches Tageblatt" vom 18.2.1956.

6 Vgl. film-dienst, Köln 9/87, S. 448.

7 Vgl. dazu auch Bernward Frank: „Kinobesuch und Fernsehnutzung“ in: Media Perspektiven $1 / 87$.

8 Vgl. dazu Horst Schäfer: Vom zweiten auf den dritten Platz. Neue Bedingungen für die nicht-gewerbliche Filmarbeit, in: Fischer Film Almanach, Frankfurt/M. 1983.

9 film-dienst, Köln 3/87, S. 140 f..

$10 \mathrm{Vgl}$. AZ München vom 29.12.1988.

11 Zitiert nach Rolf Giesen, in: „Im Hinterkopf der Tycoon, die Villa, die Starlets", in: Rheinischer Merkur/Christ und Welt, Bonn 29.7.1988, S. 17.

12 Thomas Mann: Tagebücher, S. Fischer Verlag, Frankfurt/M. 1980, 5.9.1933.

13 Vgl. dazu auch Reinhard Kleber: „Literaturverfilmung“ in: medien praxis, Frankfurt/ M. 4/87.

14 Vgl. dazu „Lexikon der Internationalen Films“ - rororo, Reinbek bei Hamburg 1988.

15 Jack Zipes: Grimms in Farbe, Bild und Ton. Der deutsche Märchenfilm für Kinder im Zeitalter der Kulturindustrie, in: Wolfgang Schneider (Hrsg.) „Aufbruch zum neuen bundesdeutschen Kinderfilm“, Eulenhof-Verlag, Hardebeck 1982, S. 213 ff..

16 Bruno Bettelheim: „Kinder brauchen Märchen“, Deutsche Verlagsanstalt, Stuttgart 1977.

17 Ergebnisse der Tagung: Märchen im Film „Vom Zauberwald zur Traumfabrik“, Kinder- und Jugendfilmkorrespondenz, München 1983.

18 Terry Gilliam in: AZ Feuilleton, München 8.12.1988, S. 18.

19 Frank Schula in: medium, Frankfurt/M. 5. Jg. Heft 12/1975. 
20 Aus: Kinder- und Jugendfilmkorrespondenz, München 3/1987, S. 24.

21 Aus: Presseheft des Filmverleihs Filmwelt, München 1982, S. 7.

22 Anne Frederiksen: Interview mit Haro Senft, in: Kinder- und Jugendfilmkorrespondenz, München 4/1987.

23 Aus: Kurzfilme im Kinderkino, in: Kinder- und Jugendfilmkorrespondenz, München Nr. 34/2/88.

\section{SUMMARY}

Children and young people have the opportunity of seeing screen adaptations of literary works in cinemas, on television and in non-commercial centres like youth and parish centres. In the Federal Republic of Germany, there are about 7.000 of these nonprofit film performance centres which, when showing certain film, are often attended by a larger audience than are the cinemas. In his article Anton Täubl analyzes aspects with regard to quantity and quality of films for children and young people, which are distributed in the Federal Republic of Germany; furthermore, he emphasizes the multiplication effect of television. By some individual examples he demonstrates especially the consistently constructive co-operation between authors of children's books and scriptwriters. Screen adaptations of literary works are frequently based on current problems of daily life and less frequently on adventure and fiction stories.

\section{RÉSUMÉ}

Les enfants et les jeunes ont la possibilité de voir des adaptations cinématographiques d'ouevres littéraires au cinéma, à la télévision et dans des établissements à caractère non commericial comme par exemple les maisons de jeunes ou les centres paroissiaux. En République fédérale d'Allemagne il y a environ 7.000 de ces lieux de projection sans but lucratif qui, lors de la présentation de certains films, comptent souvent plus de spectateurs que les cinémas. Dans son article $M$. Anton Täubl analyse des aspects concernant la quantité et la qualité des films pour enfants et jeunes gens distribués en R.F.A. et il souligne l'effet multiplicateur de la télévision. En s'appuyant sur des exemples individuels il démontre en particulier la coopération entre les auteurs de livres pour enfants et les scénaristes. Les adaptations cinématographiques d'oeuvres littéraires traitent plutût des problèmes actuels de la vie quotidienne que des aventures ou des histoires fantastiques.

\section{RESUMEN}

Los ninos y jóvenes tienen la ocasión de ver versiones cinematográficas de obras literarieas en el cine, en la televisión y en entidades no comerciales como, por ejemplo, centros parroquiales y juveniles. En la República Federal de Alemania hay aproximadamente 7000 de estos lugares de proyección de carácter no lucrativo que en cuanto a algunas películas cuentan muchas veces con más espectadores que los cines. En su arículo Anton Täubl analiza aspectos cuantitativos y cualitativos de las películas infantiles y juveniles que se distribuyen en la República Federal de Alemania. Además, destaca el efecto multiplicador de la televisión. A base de algunos ejemplos individuales demuestra en particular la colaboración generalmente constructiva entre los autores de libros para ninos y los guionistas. Las películas basadas en obras literarias tratan más bien de problemas actuales de la vida cotidiana que de aventuras o acontecimientos fantásticos. 